\title{
Concluding talk at NOW 2006
}

\author{
J. W. F. Valle ${ }^{\mathrm{a} *}$ \\ ${ }^{a}$ AHEP Group, Instituto de Física Corpuscular - C.S.I.C./Universitat de València \\ Edificio Institutos de Paterna, Apt 22085, E-46071, València, Spain
}

This writeup summarizes the status of neutrino oscillations, including recent fluxes and experimental data, as of summer 2006. A discussion is given on the current status of absolute scale of neutrino mass from tritium, $0 \nu \beta \beta$ and cosmological observations, as well as the prospects for the next generation of experiments, including lepton flavour violation searches, and their theoretical significance.

\section{Intoduction}

With almost 30 plenary talks and 70 parallel session talks the task of summarizing all of them all into a single talk is an impossible mission. I will try instead simply to highlight some aspects of the talks that touched my own prejudices.

The progress in the physics of neutrino oscillations in the last few years has been truly remarkable. Oscillations are now established, implying that neutrinos have masses, as first suggested by theorists in the early eighties, both on general grounds [1/2] and on the basis of various versions of the seesaw mechanism [3. This is a profound discovery that marks the beginning of a new age in neutrino physics.

A gold rush towards precision results has been initiated, whose aim is to probe $\theta_{13}$, to study leptonic $\mathrm{CP}$ violation and determine the nature of neutrinos. Hopefully this will shed light on the ultimate origin the universe and certainly that of neutrino mass.

\section{Data}

Thanks to the accumulation of events over a wide range of energy, and to the measurement of the dip in the $\mathrm{L} / \mathrm{E}$ (neutrino flight length $\mathrm{L}$

\footnotetext{
*Work supported by MEC grants FPA2005-01269 and FPA2005-25348-E, by Generalitat ACOMP06/154, and by the EC RTN Contract MRTN-CT-2004-503369 and ILIAS/N6 Contract RII3-CT-2004-506222. Thanks to M. Tortola, M. Maltoni and T. Schwetz.
}

over neutrino energy E) distribution of the muon neutrino disappearance probability, the interpretation of the atmospheric data has finally turned into an unambiguous signal of $\nu_{\mu} \leftrightarrow \nu_{\tau}$ oscillations, marking the beginning of a new era.

The interpretation of solar data per se is still ambiguous, with viable alternative explanations involving spin flavour precession 445 or nonstandard neutrino interactions 6]. Results on (or relevant to) solar neutrinos were presented here by Broggini, Maneira, Pulido, Raghavan, Ranucci, Serenelli and Smy. Reactor neutrino data from KamLAND not only confirm the solar neutrino deficit but also observe the spectrum distortion as expected for oscillations. Reactors have played an important role in establishing the robustness of the neutrino flavor oscillation interpretation vis a vis the existence of solar density fluctuations [7] in the solar radiative zone as produced by random magnetic fields $[8$, and also with respect to the effect of convective zone magnetic fields, should neutrinos posses nonzero neutrino transition magnetic moments [9. Within the oscillation picture KamLAND has also identified large mixing angle oscillation as its "unique" solution, "solving", in a sense, the solar neutrino problem. Note however that the interpretation of solar data vis a vis neutrino non-standard interactions [10] is not yet so robust.

A lot more is to come from the upcoming reactor experiments starting with Double-Chooz, 
and a new series of proposed experiments such as Daya-bay, RENO, Kaska, Angra, nicely reviewed in the talk by Cabrera.

Accelerators K2K \& MINOS confirm the atmospheric neutrino deficit as well as a distortion of the energy spectrum consistent with the oscillation hypothesis. More is to come from MINOS and the upcoming experiments CNGS/OPERA, T2K, NOVA, as reported here by Gugliemi, Kajita, Kato, Kopp, Rebel, Sioli, and others.

\section{Oscillation parameters}

The basic tool to interpret neutrino data is the lepton mixing matrix, whose simplest unitary 3dimensional form is given as a product of effectively complex $2 \times 2$ matrices [2]

$K=\omega_{23} \omega_{13} \omega_{12}$

where each factor is given as

$\omega_{13}=\left(\begin{array}{ccc}c_{13} & 0 & e^{i \Phi_{13}} s_{13} \\ 0 & 1 & 0 \\ -e^{-i \Phi_{13}} s_{13} & 0 & c_{13}\end{array}\right)$,

in the most convenient ordering chosen in the PDG [1] (here $c_{i j} \equiv \cos \theta_{i j}$ and $s_{i j} \equiv \sin \theta_{i j}$ ). The two Majorana phases associated to 12 and 23 can be eliminated insofar as neutrino oscillations are concerned, since they only affect leptonnumber violating processes, like $0 \nu \beta \beta$. Thus one can take the 12 and 23 factors as real, $\omega_{23} \rightarrow r_{23}$ and $\omega_{12} \rightarrow r_{12}$. There is then a unique CP phase $\Phi_{13}$, analogous to the KM phase $\delta$ of quarks, that will be studied in future oscillation experiments, such as T2K and NOVA. Currently, however, oscillations have no sensitivity to this phase and we will neglect it in the analysis of current data.

In such approximation oscillations depend on the three mixing parameters $\sin ^{2} \theta_{12}, \sin ^{2} \theta_{23}, \sin ^{2} \theta_{13}$ and on the two masssquared splittings $\Delta m_{\mathrm{SOL}}^{2} \equiv \Delta m_{21}^{2} \equiv m_{2}^{2}-m_{1}^{2}$ and $\Delta m_{\mathrm{ATM}}^{2} \equiv \Delta m_{31}^{2} \equiv m_{3}^{2}-m_{1}^{2}$ characterizing solar and atmospheric neutrinos. The fact that $\Delta m_{\mathrm{SOL}}^{2} \ll \Delta m_{\text {АтM }}^{2}$ implies that one can set $\Delta m_{\mathrm{SOL}}^{2} \rightarrow 0$ in the analysis of atmospheric and accelerator data, and $\Delta m_{\mathrm{ATM}}^{2}$ to infinity in the analysis of solar and reactor data.

\subsection{Present status}

The current three-neutrino oscillation parameters are summarized in Fig. 1. Equivalent results by the Bari group are in excellent agreement with those reported here, both pre [12] and post-MINOS [13. The analysis employs the latest Standard Solar Model [14] which we heard here in the talk by Serenelli, and includes all new neutrino oscillation data from SNO salt [15], K2K [16] and MINOS [17, described in Appendix $\mathrm{C}$ of hep-ph/0405172 (v5) 18, 2.

The upper panels of the figure show $\Delta \chi^{2}$ as a function of the three mixing parameters $\sin ^{2} \theta_{12}, \sin ^{2} \theta_{23}, \sin ^{2} \theta_{13}$ and two mass squared splittings $\Delta m_{21}^{2}, \Delta m_{31}^{2}$, minimized with respect to the undisplayed parameters. The lower panels give two-dimensional projections of the allowed regions in five-dimensional parameter space. In addition to a confirmation of oscillations with $\Delta m_{\mathrm{ATM}}^{2}$, accelerator neutrinos provide a better determination of $\Delta m_{\mathrm{ATM}}^{2}$ as one can see by comparing dashed and solid lines in Fig. 1. The recent MINOS data [17] lead to an improved determination and a slight increase in $\Delta m_{\mathrm{ATM}}^{2}$. As already mentioned, reactors 23 have played a crucial role in selecting large-mixing-angle (LMA) oscillations [24] out of the previous "zoo" of possible solar neutrino oscillation solutions 25/26. Table 1 gives the current best fit values and allowed $3 \sigma$ ranges of oscillation parameters.

Note that $\mathrm{CP}$ violation disappears in a threeneutrino scheme when two neutrinos become degenerate or when one of the angles vanishes 27 . As a result $\mathrm{CP}$ violation is doubly suppressed, first by $\alpha \equiv \Delta m_{\mathrm{SOL}}^{2} / \Delta m_{\mathrm{ATM}}^{2}$ and also by the small value of $\theta_{13}$. The left panel in Fig. 2 gives the parameter $\alpha$, while the right panel shows the impact of different data samples on constraining $\theta_{13}$.

\footnotetext{
2 In addition to good calculations of the neutrino fluxes 19/20, cross sections and response functions, we need an accurate description of neutrino propagation in the Sun and the Earth, including matter effects [21/22].
} 


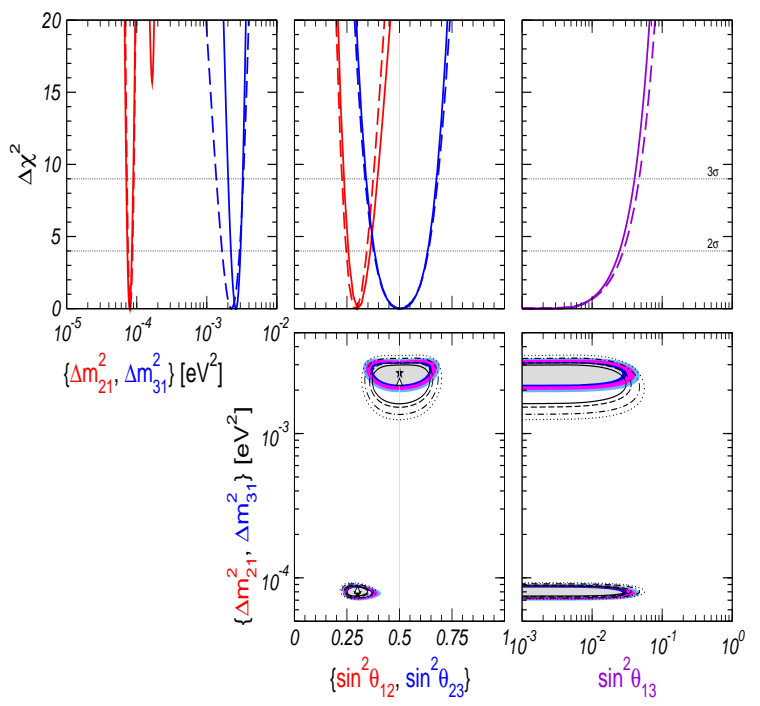

Figure 1. Current 90\%, 95\%, 99\%, and $3 \sigma$ C.L. neutrino oscillation regions for 2 d.o.f. as of summer 2006, from [18. In top panels $\Delta \chi^{2}$ is minimized with respect to undisplayed parameters.

One sees that for larger $\Delta m_{\text {ATM }}^{2}$ values the bound on $\sin ^{2} \theta_{13}$ is dominated by CHOOZ, while for low $\Delta m_{\text {AтM }}^{2}$ the solar and KamLAND data become quite relevant.

\subsection{Robustness}

Reactor neutrino data have played a crucial role in testing the robustness of solar oscillations vis a vis astrophysical uncertainties, such as magnetic fields in the radiative [78] and convective zone [5]6/4, leading to stringent limits on neutrino magnetic transition moments 9]. KamLAND has also played a key role in identifying oscillations as "the" solution to the solar neutrino problem 24] and also in pinning down the LMA parameter region among previous wide range of oscillation solutions 25]26.

However, there is still some fragility in the interpretation of the data in the presence of subweak strength $\left(\sim \varepsilon G_{F}\right)$ non-standard neutrino interactions (NSI) (Fig. 3). Indeed, most neu-

\begin{tabular}{|l|c|c|}
\hline parameter & best fit & $3 \sigma$ range \\
\hline \hline$\Delta m_{21}^{2}\left[10^{-5} \mathrm{eV}^{2}\right]$ & 7.9 & $7.1-8.9$ \\
$\Delta m_{31}^{2}\left[10^{-3} \mathrm{eV}^{2}\right]$ & 2.6 & $2.0-3.2$ \\
$\sin ^{2} \theta_{12}$ & 0.30 & $0.24-0.40$ \\
$\sin ^{2} \theta_{23}$ & 0.50 & $0.34-0.68$ \\
$\sin ^{2} \theta_{13}$ & 0.00 & $\leq 0.040$ \\
\hline
\end{tabular}

Table 1

Neutrino oscillation parameters as of Summer 2006, from Ref. [18].
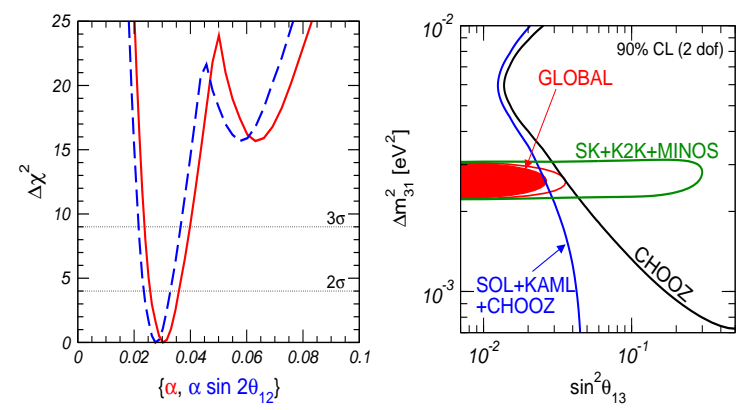

Figure 2. $\alpha \equiv \Delta m_{\mathrm{SOL}}^{2} / \Delta m_{\mathrm{ATM}}^{2}$ and $\sin ^{2} \theta_{13}$ bound from the updated analysis given in Ref. [18].

trino mass generation mechanisms imply the existence of such dimension- 6 operators. They can be of two types: flavour-changing (FC) and nonuniversal (NU). Their presence leads to the possibility of resonant neutrino conversions even in the absence of neutrino masses [28. While modeldependent, the expected NSI magnitudes may well fall within the range that will be tested in future precision studies [29]. For example, in the inverse seesaw model 30 the non-unitary piece of the lepton mixing matrix can be sizeable, hence the induced non-standard interactions. Relatively sizeable NSI strengths may also be induced in supersymmetric unified models 31 and models with radiatively induced neutrino masses [32 33.

The determination of atmospheric neutrino parameters $\Delta m_{\text {ATM }}^{2}$ and $\sin ^{2} \theta_{\text {ATM }}$ is hardly affected by the presence of NSI on down-type quarks [34]. 


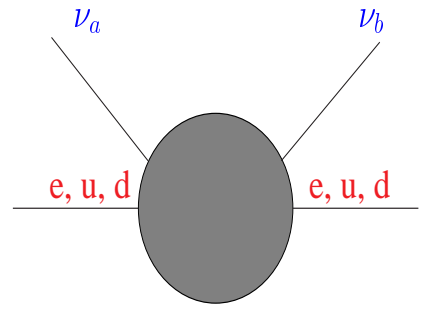

Figure 3. Non-standard neutrino interactions arise, e. g., from the non-unitary structure of charged current weak interactions characterizing seesaw-type schemes [2].

In contrast, the determination of solar neutrino parameters is not quite robust against the existence of NSI 10, even if reactor data are included. The issue can only be resolved by future low and intermediate energy solar neutrino data mentioned by Raghavan, with enough precision to sort out the detailed profile of the solar neutrino conversion probability.

\subsection{Future prospects}

Upcoming reactor and accelerator long baseline experiments aim at improving the sensitivity on $\sin ^{2} \theta_{13}$ 29. The value of $\theta_{13}$ is a key input and the start of an ambitious long-term effort towards probing $\mathrm{CP}$ violation in neutrino oscillations 35 36 37. Prospects of accelerator and reactor neutrino oscillation experiments for the coming years have been extensively discussed in the literature and there have been several talks at this conference, for example those of Declais, Huber, Kajita, Kato, Kopp, Lindner, Nunokawa and Schwetz. Here I simply illustrate in Fig. 4 the anticipated evolution of the $\theta_{13}$ discovery reach for the global neutrino program, see details in Ref. 38 .

One important comment is that even a small residual non-standard interaction in this "solar" (e-tau) channel has dramatic consequences for the sensitivity loss for $\theta_{13}$ at a neutrino factory 39 . To make these experiments meaningful it is a

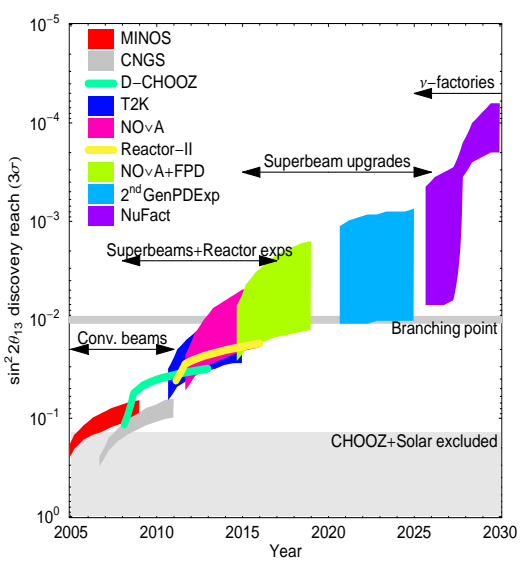

Figure 4. The hunt for $\theta_{13}$ : artist's view of anticipated sensitivities on $\theta_{13}$ given in Ref. [38].

must to have a near detector capable of sorting out for NSI with high sensitivity.

In contrast, future neutrino factories will probe flavor changing non-standard neutrino-matter interactions in the "atmospheric" (mu-tau) channel with sensitities which are substantially improved with respect to current ones 40. For example, a $100 \mathrm{GeV}$ NUFACT can probe these at the level of $|\epsilon|<$ few $\times 10^{-4}$ at $99 \%$ C.L.

Improving the sensitivities on NSI constitutes at a near detector is a necessary pre-requisit. In short, probing for NSI is an important item and a window of opportunity for neutrino physics in the precision age.

To close this section let me mention that day/night effect studies in large water Cerenkov solar neutrino experiments such as UNO, Hyper$\mathrm{K}$ or LENA has also been suggested as an alternative way to probe $\theta_{13}$ [41.

\section{Lepton flavour violation}

The discovery of neutrino oscillations demonstrates that lepton flavour conservation is not a fundamental symmetry of nature. It is therefore 
natural to expect that it may show up elsewhere, for example $\mu \rightarrow e \gamma$ or nuclear $\mu^{-}-e^{-}$conversion, as seen in Fig. 5. Indeed, in seesaw-type

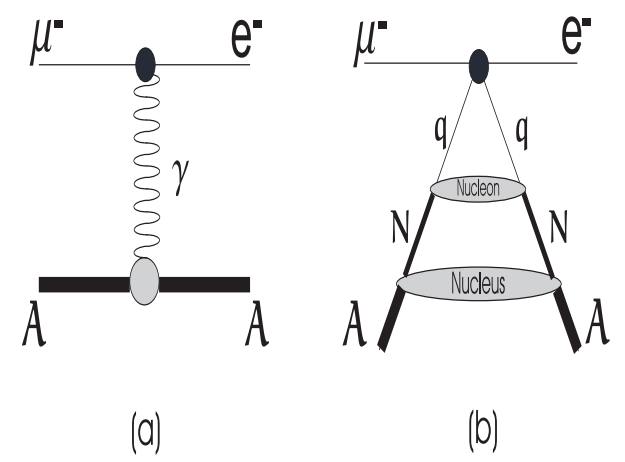

Figure 5. Contributions to the nuclear $\mu^{-}-$ $e^{-}$conversion: (a) long-distance and (b) shortdistance. For numerical results see Ref. 30]42]

schemes of neutrino mass, lepton flavour violation is induced either from neutral heavy lepton exchange 43144 as discussed here by Vogel, or through the exchange of charginos (neutralinos) and sneutrinos (charged sleptons) as discussed here by Masiero 45/46/47/48] (see top panel in Fig. 6). As illustrated in Fig. 6 the rates for both processes can be sizeable and fall within the sensitivity of upcoming experiments. The calculation in Fig. [6] is performed in the framework of the generalized supersymmetric seesaw model of Ref. 30. to where I address you if you wish to understand the interplay of neutral heavy lepton [43] and supersymmetric contributions. If the neutral heavy leptons are in the $\mathrm{TeV}$ range (a situation not realizable in the minimal seesaw mechanism), the $B r(\mu \rightarrow e \gamma)$ rate can be enhanced even in the $a b-$ sence of supersymmetry. In this case the neutral heavy leptons that mediate lepton flavour violation may be directly produced at accelerators [4].

Fig. 6 also illustrates the correlation between nuclear $\mu^{-}-e^{-}$conversion and $\mu^{-} \rightarrow e^{-} \gamma$ decay
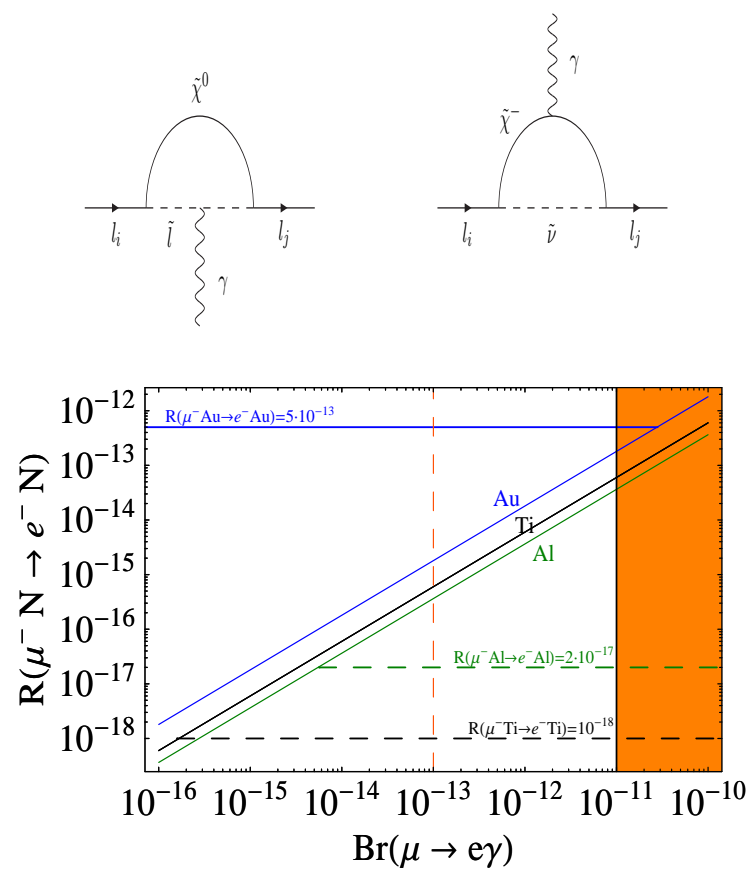

Figure 6. Supersymmetric Feynman diagrams for lepton flavour violation and correlation between mu-e conversion and $\operatorname{Br}(\mu \rightarrow e \gamma)$ in the supersymmetric inverse seesaw model of Ref. [30].

in the inverse seesaw model for the nuclei $\mathrm{Au}$, $\mathrm{Ti}, \mathrm{Al}$. The shaded area and vertical line denote the current bound and future sensitivity (PSI) on $\operatorname{Br}(\mu \rightarrow e \gamma)$, respectively. The horizontal lines denote the current bound (Au/SINDRUM II) and expected future sensitivities (Al/MECO, Ti/PRISM) on $R\left(\mu^{-} N \rightarrow e N\right)$. More details in Ref. [42.

Note also that since lepton flavour violation and $\mathrm{CP}$ violation can occur in the massless neutrino limit, hence the allowed rates need not be suppressed by the smallness of neutrino masses [3]44|50|51].

\section{Absolute scale of neutrino mass}

Neutrino oscillations are insensitive to absolute masses and can not probe whether neutrinos are Dirac or Majorana. Current data can not de- 
termine whether the spectrum is normal or inverted, as illustrated in Fig. 7 To settle the is-

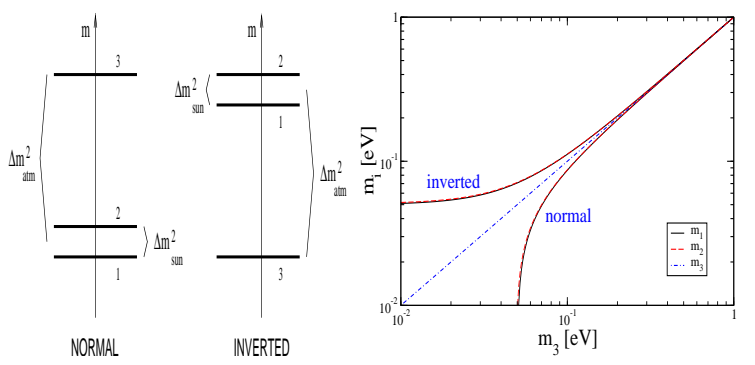

Figure 7. Which spectrum?

sue one needs kinematical tests, such as beta decay studies [52, as discussed here by Sisti and Weinheimer. The upcoming high precision neutrino mass experiment KATRIN scales up both the size \& source intensity, aiming at a sensitivity one order of better than that of the current Mainz-Troitsk experiments.

Neutrinoless double beta decay and other lepton number violation processes, such as neutrino transition electromagnetic moments $53 \mid 54$ 55156] can probe the basic nature of neutrinos.

The significance of neutrinoless double beta decay stems from the fact that, in a gauge theory, irrespective of the mechanism that induces $0 \nu \beta \beta$, it necessarily implies a Majorana neutrino mass [57, as illustrated in Fig. 8. Thus it is a basic issue. Quantitative implications of the "black-box" argument are model-dependent, but the theorem itself holds in any "natural" gauge theory.

Now that oscillations are experimentally confirmed we know that $0 \nu \beta \beta$ must be induced by the exchange of light Majorana neutrinos, the so-called "mass-mechanism". The $0 \nu \beta \beta$ amplitude depends on the 3 masses, 2 mixing angles, and $2 \mathrm{CP}$ phases. Hence it involves the absolute scale of neutrino mass, as well as the Majorana phase [2, neither of which can be probed

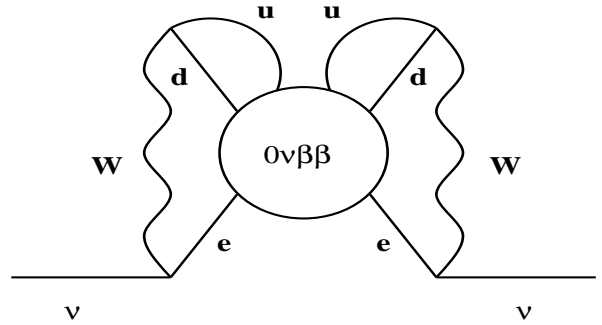

Figure 8. Neutrinoless double beta decay and Majorana mass are equivalent [57].

in oscillations [58/5960]. The phenomenological situation was described here by Avignone, Bettini, Fiorini, Pavan, Simkovik, Vala and Vogel. It clearly distinguishes between normal and inverted hierarchical spectra, as seen in Fig. 9, in the former hierarchy case there is in general no lower bound on the $0 \nu \beta \beta$ rate, since there can be a destructive interference amongst the neutrino amplitudes. In contrast, the inverted neutrino mass hierarchy implies a "lower" bound for the $0 \nu \beta \beta$ amplitude. The best current limit on $\left\langle m_{\nu}\right\rangle$ comes from the Heidelberg-Moscow experiment. The current claim [62 (see also Ref. 63]) and the sensitivities of the upcoming experiments are indicated in the compilation, courtesy of Simkovik, displayed in Fig. 9. It shows the estimated average mass parameter characterizing the neutrino exchange contribution to $0 \nu \beta \beta$ versus the lightest and heaviest neutrino masses. The calculation takes into account the current neutrino oscillation parameters in [18] and state-of-the-art nuclear matrix elements 61]. The upper (lower) panel corresponds to the cases of normal (inverted) neutrino mass spectra. In these plots the "diagonals" correspond to the case of quasi-degenerate neutrinos 64 65, 66.

We now give two examples of model $0 \nu \beta \beta$ expectations. First, Ref. 67 proposes a specific normal hierarchy model for which a lower bound on $0 \nu \beta \beta$ can be placed, as a function of the value of the Majorana violating phase $\phi_{1}$, as indicated 

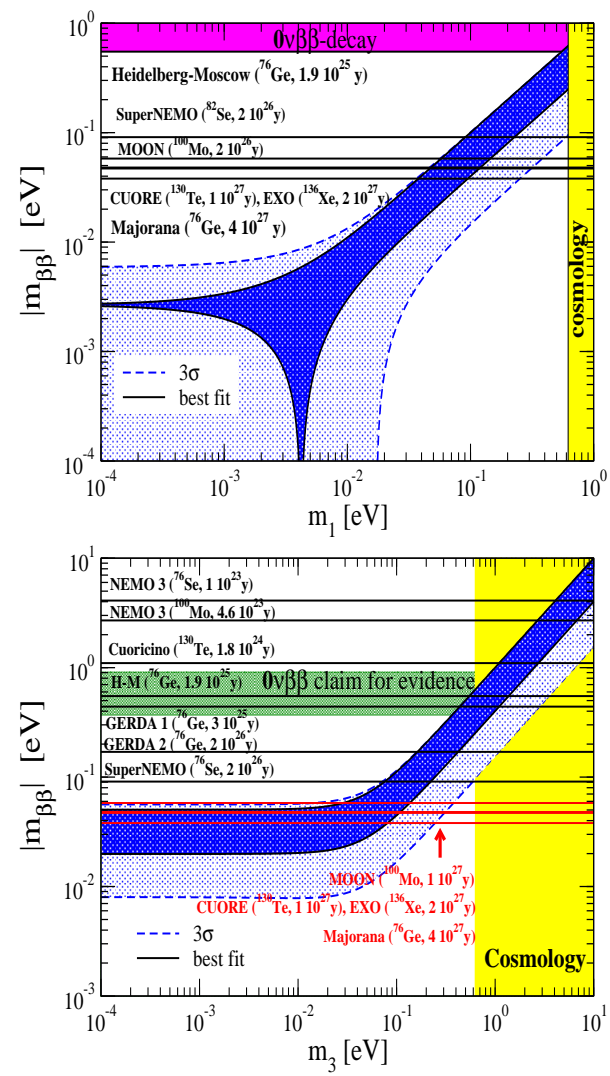

Figure 9. $0 \nu \beta \beta$ amplitude versus current oscillation data, from Ref. [61.

in Fig. 10. Second, the $A_{4}$ model 64] gives a lower bound on the absolute Majorana neutrino mass $m_{\nu} \gtrsim 0.3 \mathrm{eV}$ and may therefore be tested in $0 \nu \beta \beta$ searches.

The absolute scale of neutrino masses will be tested by its effect on the cosmic microwave background and the large scale structure of the Universe, as discussed here by Elgaroy, Palazzo, Pastor and Viel 68|69|13], and illustrated in Fig. 11,

\section{Neutrinos as probes}

Not only neutrino properties can be probed via cosmology and astrophysics, but also, once

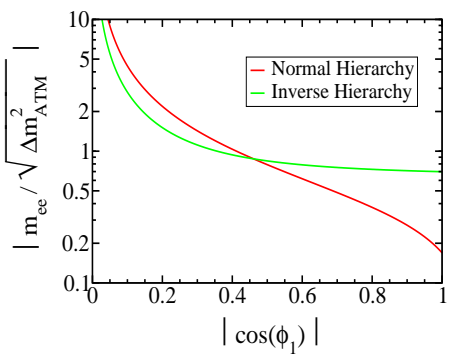

Figure 10. Lower bound on $\left|\left\langle m_{e e}\right\rangle\right| / \Delta m_{\text {ATM }}^{2}$ vs $\left|\cos \left(\phi_{1}\right)\right|$ where $\phi_{1}$ is a Majorana phase. The lines in dark (red) and grey (green) correspond to normal and inverse hierarchy. Model of Ref. 67.

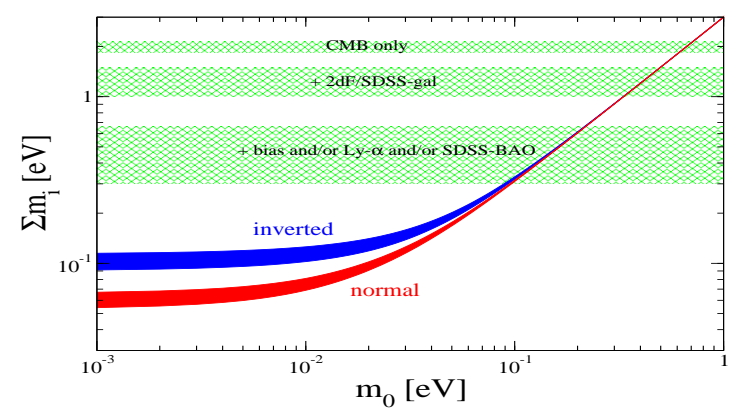

Figure 11. Sensitivity of cosmology to neutrino mass, see Ref. [68.

well-determined, they can be used as astro-probes (Sun, Supernovae, pulsars, etc), cosmo-probes even geo-probes. Here there were many related talks by Elgaroy, Mangano, Pastor, Villante, and Viel. Neutrinos affect nucleosynthesis, large scale structure, the CMB and possibly the generation of the matter anti-matter asymmetry.

Like photons, cosmic rays and gravitational waves, neutrinos are one of the basic messengers of the Big Bang capable of probing early stages of its evolution. In the leptogenesis scenarios (discussed here by Akhmedov, di Bari, Ma and Petcov) neutrinos could probe the Universe even 
down to epochs prior to the electroweak phase transition.

Neutrinos are also basic probes is astrophysics. Having only weak interactions, they are ideal to monitor the interior of stars, such as the the Sun.

Supernova neutrinos were discussed by Cardall, Fleurot, Lunardini and Vagins. The measurement of a large number of neutrinos from a future galactic supernova neutrino signal will give us important information on supernova parameters. Here I give a simplified plot (small $\theta_{13}$ approximation) taken from Ref. 70 as an illustration of the potential to probe astrophysics from a precise knowledge of neutrino properties.
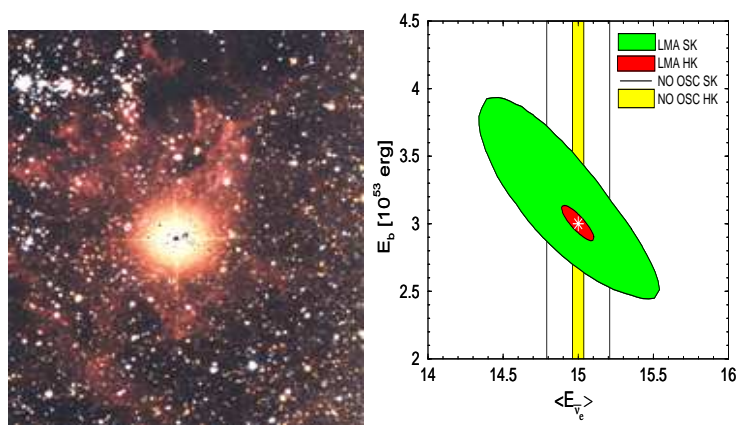

Figure 12. Improved supernova parameter determination attainable from the neutrino signal from $10 \mathrm{kpc}$ galactic supernova within future neutrino telescopes Hyper-K versus Super-K.

Let me also mention that new effects in neutrino conversions at the core of supernovae (neutron-rich regime) are expected when neutrinos have non-standard interactions $28 / 71 / 72$. These could induce new inner resonant conversions, over and above those that arise from oscillations.

Before closing this section, let me mention that neutrinos are ideal probes of the high energy Universe, discussed here by Billoir, Karle, Flaminio, Stanev and Sigl. For example, one expects high energy neutrinos from AGNs and GRBs. Typi- cally the accelerated primaries make pions, leading to comparable fluxes of neutrinos and gammas due to isospin. In contrast to gamma-rays, the neutrino spectrum is essentially unmodified. One set of observables to monitor are the flavor ratios, which are sensitive both to neutrino oscillations as well as neutrino non-standard interactions.

Here I give a very useful roadmap-plot presented by Sigl (see his talk at these proceedings for details). This plot makes extrapolations as to what high energy neutrino fluxes could be on the basis of the gamma-flux constraints (e. g. by EGRET) at lower energies.

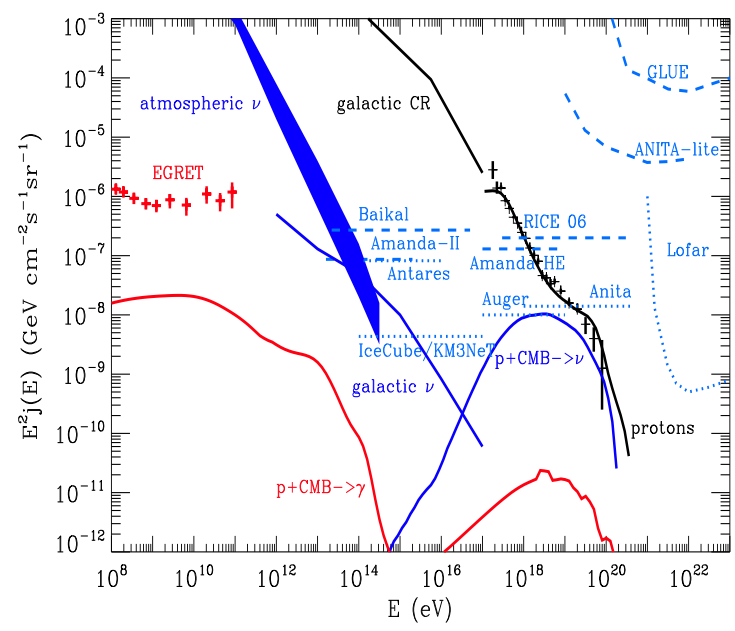

Figure 13. Sigl's map to high energy astrophysics.

\section{Origin of neutrino mass}

This is one of the most well-kept secrets of nature. Gauge theories prefer Majorana neutrinos (see historical talk by Esposito) [12] irrespective the detailed mechanism of neutrino mass generation. While possible, the emergence of Dirac neutrinos would constitute a surprise, indicating the existence of an accidental lepton number symmetry whose fundamental origin should be understood. There are some ideas for generating 
light Dirac neutrinos. For example, theories involving large extra dimensions offer a novel scenario to account for small Dirac neutrino masses. Within this picture, right-handed neutrinos propagate in the bulk, while left-handed neutrinos, being a part of the lepton doublet, live only on the Standard Model (SM) branes. In this picture neutrinos get naturally small Dirac masses via mixing with a bulk fermion.

However neutrinos are more likely Majorana.

In contrast to SM charged fermions, neutrinos do not get masses after the electroweak symmetry breaks through by the nonzero vacuum expectation value (vev) of the Higgs scalar doublet, since they come in just one chiral species. There is, however, an effective lepton number violating dimension-five operator $L L \Phi \Phi$ (where $\mathrm{L}$ denotes any of the lepton doublets and $\Phi$ the Higgs) which can be added to the SM [1]. After the Higgs mechanism this operator induces Majorana neutrino masses, thus providing a natural way to account for their smallness, irrespective of their specific origin. Little more can be said from first principles about the mechanism giving rise to this operator, its associated mass scale or its flavour structure. Its strength may be suppressed by a large scale in the denominator (top-down) scenario, as in seesaw schemes [3. Alternatively, the strength may be suppressed by small parameters (e.g. scales, Yukawa couplings) and/or loopfactors (bottom-up scenario) with no need for a large scale, as also reviewed in Ref. 3].

\section{REFERENCES}

1. S. Weinberg, Phys. Rev. D22, 1694 (1980).

2. J. Schechter and J. W. F. Valle, Phys. Rev. D22, 2227 (1980); Phys. Rev. D 25, 774 (1982).

3. For a recent seesaw review see J. W. F. Valle, lectures at the Corfu Summer Institute on Elementary Particle Physics hep-ph/0608101.

4. J. Barranco et al, Phys. Rev. D66, 093009 (2002)

5. O. G. Miranda et al., Nucl. Phys. B595, 360
(2001), hep-ph/0005259.

6. M. Guzzo et al, Nucl. Phys. B629, 479 (2002)

7. C. P. Burgess et al., JCAP 0401, 007 (2004); Astrophys J 588 L65 (2003)

8. C. P. Burgess et al, Mon. Not. Roy. Astron. Soc. 348, 609 (2004)

9. O. G. Miranda et al, Phys. Rev. Lett. 93 051304 (2004); Phys. Rev. D70 113002 (2004)

10. O. G. Miranda, M. A. Tortola and J. W. F. Valle, JHEP 10, 008 (2006)

11. Particle Data Group, W. M. Yao et al., J. Phys. G33, 1 (2006).

12. G. L. Fogli, E. Lisi, A. Marrone, A. Palazzo, Prog. Part. Nucl. Phys. 57 (2006) 742

13. G. L. Fogli et al., hep-ph/0608060.

14. J. N. Bahcall, A. M. Serenelli and S. Basu, astro-ph/0511337.

15. SNO collaboration, B. Aharmim et al., Phys. Rev. C72, 055502 (2005)

16. K2K collaboration, M. H. Ahn, Phys. Rev. D74, 072003 (2006)

17. D. G. Michael et al. [MINOS Collaboration], Phys. Rev. Lett. 97 (2006) 191801

18. M. Maltoni et al., New J. Phys. 6, 122 (2004), Appendix C in hep-ph/0405172 (v5) provides updated results as of Summer 2006; previous references to other groups therein.

19. J. N. Bahcall and M. H. Pinsonneault, Phys. Rev. Lett. 93, 121301 (2004)

20. M. Honda, T. Kajita, K. Kasahara and S. Midorikawa, astro-ph/0404457.

21. S. P. Mikheev and A. Y. Smirnov, Sov. J. Nucl. Phys. 42, 913 (1985).

22. L. Wolfenstein, Phys. Rev. D17, 2369 (1978).

23. KamLAND collaboration, T. Araki et al., Phys. Rev. Lett. 94, 081801 (2004).

24. S. Pakvasa and J. W. F. Valle, hep-ph/0301061, Proc. Indian National Acad. of Sci., Vol. 70A, No.1, p.189 - 222 (2004)

25. M. C. Gonzalez-Garcia et al, Phys. Rev. D63, 033005 (2001)

26. G. L. Fogli, E. Lisi, D. Montanino and A. Palazzo, Phys. Rev. D64, 093007 (2001)

27. J. Schechter and J. W. F. Valle, Phys. Rev. 
D21, 309 (1980).

28. J. W. F. Valle, Phys. Lett. B199, 432 (1987).

29. P. Huber, M. Lindner, Rolinec, T. Schwetz, W. Winter, Phys. Rev. D70 073014 (2004)

30. F. Deppisch and J. W. F. Valle, Phys. Rev. D72, 036001 (2005), hep-ph/0406040].

31. L. J. Hall, V. A. Kostelecky and S. Raby, Nucl. Phys. B267, 415 (1986).

32. A. Zee, Phys. Lett. B93, 389 (1980).

33. K. S. Babu, Phys. Lett. B203, 132 (1988).

34. N. Fornengo et al., Phys. Rev. D65, 013010 (2002); For 3-neutrino case see A. Friedland, C. Lunardini and M. Maltoni, Phys. Rev. D 70 (2004) 111301

35. Muon Collider/Neutrino Factory, M. M. Alsharoa et al., Phys. Rev. ST Accel. Beams 6, 081001 (2003), hep-ex/0207031.

36. M. Apollonio et al., hep-ph/0210192, CERN Yellow Report on the Neutrino Factory.

37. C. Albright et al., hep-ex/0008064, Report to the Fermilab Directorate.

38. M. G. Albrow et al., hep-ex/0509019.

39. P. Huber, T. Schwetz and J. W. F. Valle, Phys. Rev. Lett. 88, 101804 (2002); Phys. Rev. D66, 013006 (2002)

40. P. Huber and J. W. F. Valle, Phys. Lett. B523, 151 (2001), hep-ph/0108193.

41. E. K. Akhmedov, M. A. Tortola and J. W. F. Valle, JHEP 05, 057 (2004)

42. F. Deppisch, T. S. Kosmas and J. W. F. Valle, Nucl. Phys. B 752 (2006) 80

43. J. Bernabeu et al., Phys. Lett. B187, 303 (1987); M. C. Gonzalez-Garcia and J. W. F. Valle, Mod. Phys. Lett. A7, 477 (1992).

44. A. Ilakovac and A. Pilaftsis, Nucl. Phys. B437, 491 (1995), hep-ph/9403398.

45. L. J. Hall, V. A. Kostelecky and S. Raby, Nucl. Phys. B267, 415 (1986).

46. F. Borzumati and A. Masiero, Phys. Rev. Lett. 57, 961 (1986).

47. J. A. Casas and A. Ibarra, Nucl. Phys. B618, 171 (2001), hep-ph/0103065.

48. S. Antusch, E. Arganda, M. J. Herrero and A. Teixeira, hep-ph/0607263.

49. M. Dittmar et al., Nucl. Phys. B332, 1
(1990); P. Abreu et al. [DELPHI Collab], Z. Phys. C 74 (1997) 57 \& C 75 (1997) 580].

50. G. C. Branco, M. N. Rebelo and J. W. F. Valle, Phys. Lett. B225, 385 (1989).

51. N. Rius and J. W. F. Valle, Phys. Lett. B246, 249 (1990).

52. KATRIN collaboration, G. Drexlin, Nucl. Phys. Proc. Suppl. 145, 263 (2005).

53. J. Schechter and J. W. F. Valle, Phys. Rev. D24, 1883 (1981), Err. D25, 283 (1982).

54. L. Wolfenstein, Phys. Lett. B107, 77 (1981).

55. P. B. Pal and L. Wolfenstein, Phys. Rev. D25, 766 (1982).

56. B. Kayser, Phys. Rev. D26, 1662 (1982).

57. J. Schechter and J. W. F. Valle, Phys. Rev. D25, 2951 (1982).

58. S. M. Bilenky, J. Hosek and S. T. Petcov, Phys. Lett. B94, 49 (1980).

59. J. Schechter and J. W. F. Valle, Phys. Rev. D23, 1666 (1981).

60. M. Doi et al.,Phys. Lett. B102, 323 (1981).

61. S. M. Bilenky, A. Faessler and F. Simkovic, Phys. Rev. D70, 033003 (2004)

62. H. V. Klapdor-Kleingrothaus et al. Phys. Lett. B586, 198 (2004), hep-ph/0404088.

63. C. E. Aalseth et al., Mod. Phys. Lett. A17, 1475 (2002), hep-ex/0202018.

64. K. S. Babu, E. Ma and J. W. F. Valle, Phys. Lett. B552, 207 (2003), hep-ph/0206292.

65. D. O. Caldwell and R. N. Mohapatra, Phys. Rev. D48, 3259 (1993).

66. A. Ioannisian and J. W. F. Valle, Phys. Lett. B332, 93 (1994), hep-ph/9402333.

67. M. Hirsch et al., Phys. Rev. D72, 091301 (2005), hep-ph/0507148].

68. J. Lesgourgues and S. Pastor, Phys. Rep. 429, 307 (2006), astro-ph/0603494.

69. S. Hannestad, hep-ph/0602058.

70. H. Minakata, H. Nunokawa, R. Tomas and J. W. F. Valle, Phys. Lett. B542, 239 (2002)

71. H. Nunokawa et al., Phys. Rev. D54, 4356 (1996), hep-ph/9605301.

72. H. Nunokawa, A. Rossi and J. W. F. Valle, Nucl. Phys. B482, 481 (1996) 